International Journal of Pure and Applied Mathematics

Volume 109 No. 4 2016, 1029-1054

ISSN: 1311-8080 (printed version); ISSN: 1314-3395 (on-line version)

url: http://www.ijpam.eu

doi: 10.12732/ijpam.v109i4.21

ijpam.eu

\title{
A CONTINUOUS-TIME MARKOV CHAIN APPROACH TO FINANCIAL MODELLING: ANALYSIS AND APPLICATIONS
}

\author{
Mattia Turra \\ Department of Computer Science \\ University of Verona \\ Strada le Grazie, 15-37134, Verona, ITALY
}

\begin{abstract}
The present paper provides a continuous-time Markov chain (CTMC) model approach to financial markets. Sufficient conditions which guarantee the absence of arbitrage and the completeness of rather generic market models, aro also derived.

Then, by proving a weak converge result, we show that the CTMC model can be viewed as a generalization of the geometric Poisson model, the Black-Scholes model and the Merton model.
\end{abstract}

AMS Subject Classification: 60H15, 60H35, 91B60, 91G20, 91G60

Key Words: continuous-time Markov chain, geometric Brownian motion, compound Poisson process, geometric Poisson process, weak convergence, market modeling, finance

\section{Introduction}

In the mathematical finance scenario, a large quantity of models have been proposed aiming at describing the price process of assets, liabilities, derivatives, etc. A turning point is certainly represented by the work of Robert Merton, see [22], who exploited stochastic calculus techniques to rigorously study some relevant financial problems, in 1969. Meanwhile, Fischer Black and Myron Scholes were developing their acclaimed article, The pricing of options and corporate liabilities, see [14], published in 1973, where they used a geometric Brownian motion to model the price of a stock asset, also providing their celebrated option pricing formula. In 1976, with the paper Option pricing when underlying

$\begin{array}{lr}\text { Received: } & \text { September 6, } 2016 \\ \text { Revised: } & \text { October 5, 2016 } \\ \text { Published: } & \text { October 9, } 2016\end{array}$

(c) 2016 Academic Publications, Ltd. url: www.acadpubl.eu 
stock returns are discontinuous [23], Robert Merton developed a new model that, for the first time, takes into account a jump component, allowing to represent particular events able to influence the market by drastically changing the value of one or more assets. This model is, in fact, a particular case of a market where stocks are driven by both a geometric Brownian motion and a compound Poisson process, see, e.g., [28, Ch. 11]. Further developments in describing the increasing complexity of financial markets have been also produced exploiting different approaches, namely by looking at more general type of (systems of) stochastic (partial) differential equations, see, e.g., [4, 21, 10, 11, 12], different (namely, more general) type of stochastic processes for particular forecasting pourposes, see, e.g., [18] in the case of energy markets, or explicitly taking into account the network nature of interconnected modern economic markets also in connection with default phenomena, see, e.g., [6, 19] and references therein, etc. From another point of view, deeper insight have been reached particularly with respect to the statistical analysis of financial time series, mainly aiming at forecasting particular turbulent scenarios, as the ones realised during recent financial crisis, see, e.g., [16, 17], and references therein. More recently, Ragnar Norberg proposed, see [25], a continuous-time Markov chain (CTMC) market model, that appears to be as a generalisation of the classical approaches developed by Black, Scholes and Merton. In particular, in such a model stock price processes depend on the stochastic process that counts the number of transition from a particular state to another, the latter representing the states of the economy regarded as the components of an $n$-dimensional continuous-time homogeneous Markov chain. The present work is so structured: in Sec. 2, we introduce the CTMC model; in Sec. 3 we recall the results obtained by Norberg in [25]; eventually, in Sec. 4, we prove a weak convergence result which clearly states the connection between the Norberg model and both the Poisson market model, the Black-Scholes model, and the Merton model.

\section{The Market Model}

We start giving a brief review about the market model introduced by Norberg in $[25]$. Let $(\Omega, \mathcal{F}, \mathbb{P})$ be a probability space. Let us consider a $n$-dimensional and homogeneous continuous-time Markov chain (CTMC) $Y=\left\{Y_{t}\right\}_{t \geq 0}$, whose states represents the states of the economy. We denote the finite state space by $\mathcal{Y} \doteq\{1, \ldots, n\}$. We remind the definition of the transition intensities of the 
chain, namely

$$
\begin{aligned}
\lambda_{i} & \doteq \lim _{t \rightarrow 0} \frac{1-p_{i i}(t)}{t}, \quad i \in \mathcal{Y}, \\
\lambda_{i j} & \doteq \lim _{t \rightarrow 0} \frac{p_{i j}(t)}{t}, \quad i, j \in \mathcal{Y},
\end{aligned}
$$

both the limits exist assuming that, for all $i, j \in \mathcal{Y}$,

$$
p_{i j}(0)=\lim _{t \rightarrow 0^{+}} p_{i j}(t)=\delta_{i j}
$$

where $\delta_{i j}$ is the Kronecker delta, see, e.g., [5, Sec. 5.2]. In particular we include the conditional transition intensities $\lambda_{i j}, i, j \in \mathcal{Y}$, in the infinitesimal matrix $\Lambda=\left(\lambda_{i j}\right)_{i, j \in \mathcal{Y}}$.

We assume that all states intercommunicate, so that $p_{j k}(t)>0$, for all $j, k \in \mathcal{Y}$ and $t>0$, hence we do not have absorbing states. We define the set of states that are accessible from $j \in \mathcal{Y}$ by $\mathcal{Y}_{j} \doteq\left\{k \in \mathcal{Y}: \lambda_{j k}>0\right\}$.

Let us define two stochastic processes, based on the CTMC, that will be used in the following. The indicator process $I^{j}=\left\{I_{t}^{j}\right\}_{t \geq 0}, j \in \mathcal{Y}$, which indicates that $Y$ is in state $j$ at time $t$, namely it is defined by

$$
I_{t}^{j} \doteq \mathbb{1}_{Y_{t}=j}
$$

The counting process $N^{j k}=\left\{N_{t}^{j k}\right\}_{t \geq 0}, j, k \in \mathcal{Y}$, which counts the number of transitions from state $j$, to state $k$, in the time interval $[0, t]$, hence it is defined by

$$
N_{t}^{j k} \doteq\left|\left\{s \in(0, t]: Y_{s^{-}}=j, Y_{s}=k\right\}\right|,
$$

if $k \notin \mathcal{Y}_{j}$, then $N_{t}^{j k} \equiv 0$. We assume that $Y$ is right-continuous, so that both $I^{j}$ and $N^{j k}$ are right-continuous, too.

The state process $Y$, the indicator processes and the counting processes carry the same information, which is represented, at any time $t$, by the $\sigma$ algebra $\mathcal{F}_{t}^{Y} \doteq \sigma\left(\left\{Y_{s}: 0 \leq s \leq t\right\}\right)$. The corresponding filtration, denoted by $\mathbf{F}^{Y} \doteq\left\{\mathcal{F}_{t}^{Y}\right\}_{t \geq 0}$, is defined as the standard one, namely it satisfies the usual conditions of right-continuity and completeness, furthermore, $\mathcal{F}_{0}$ is assumed to be the trivial $\{\emptyset, \Omega\}$. Therefore, $Y$ is right-continuous and $Y_{0}$ is deterministic.

Note that $\left\{I_{t}^{j} \lambda_{j k}\right\}_{t \geq 0}$ is the intensity process of $N^{j k}, j, k \in \mathcal{Y}$, thus, the compensated counting processes $M^{j k}=\left\{M_{t}^{j k}\right\}_{t \geq 0}, j, k \in \mathcal{Y}, j \neq k$, defined by

$$
d M_{t}^{j k}=d N_{t}^{j k}-I_{t}^{j} \lambda_{j k} d t
$$


and $M_{0}^{j k}=0$, are zero mean, square integrable, mutually orthogonal martingales with respect to $\left(\mathbf{F}^{Y}, \mathbb{P}\right)$, as a byproduct of the Doob-Meyer decomposition theorem, see, e.g., [29, Sec. 12.11].

In what follows, $Y_{t}$ will represent the state of the economy at a given time $t$, while $\mathcal{F}_{t}^{Y}$ represents the information available about the market history up to the same time, and $\mathbf{F}^{Y}$ takes into account the flow of such information over time.

Let us consider $m+1$ basic assets, which can be traded freely and ifrictionlessly. Suppose that the asset number 0 is a locally risk-free bank account with state dependent interest rate

$$
r_{t}=r^{Y_{t}}=\sum_{j=1}^{n} I_{t}^{j} r^{j}
$$

where the state-wise interest rates $r^{j}, j \in \mathcal{Y}$, are constants. Thus, its price process is given by

$$
S_{t}^{0}=S_{0}^{0} \exp \left(\int_{0}^{t} r_{s} d s\right)=S_{0}^{0} \exp \left(\sum_{j=1}^{n} r^{j} \int_{0}^{t} I_{s}^{j} d s\right),
$$

with dynamics

$$
d S_{t}^{0}=S_{t}^{0} r_{t} d t=S_{t}^{0} \sum_{j=1}^{n} r^{j} I_{t}^{j} d t
$$

The remaining $m$ assets, that we refer to as stocks, are risky and their price process reads as follows

$$
S_{t}^{i}=S_{0}^{i} \exp \left(\sum_{j=1}^{n} \alpha_{i j} \int_{0}^{t} I_{s}^{j} d s+\sum_{j=1}^{n} \sum_{k \in \mathcal{Y}_{j}} \beta_{i j k} N_{t}^{j k}\right)
$$

$i=1, \ldots, m$, where $\alpha_{i j}$ and $\beta_{i j k}$ are constants and, for each $i$, at least one of the $\beta_{i j k}$ is non-null. A trajectory example of an asset with price process as in equation (2) is shown in Figure 1.

Applying the Itô-Doeblin formula for jump processes to $S_{t}^{i}$ defined in eq. (2), we obtain

$$
d S_{t}^{i}=S_{t^{-}}^{i}\left(\sum_{j=1}^{n} \alpha_{i j} I_{t}^{j} d t+\sum_{j=1}^{n} \sum_{k \in \mathcal{Y}_{j}} \gamma_{i j k} d N_{t}^{j k}\right), \quad i=1, \ldots, m
$$




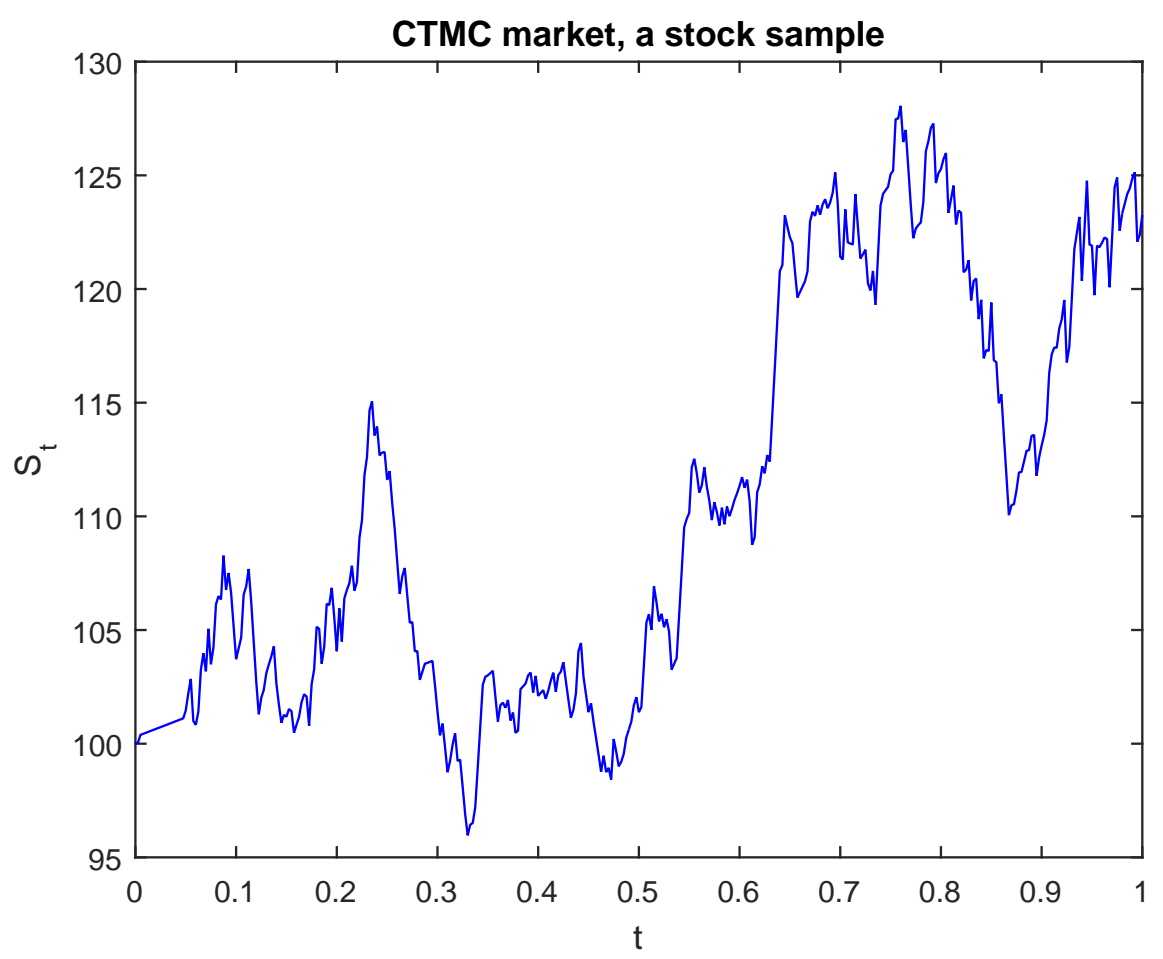

Figure 1: A sample-trajectory of an asset price following equation (2), with $S_{0}=100$ and $T=1$. The coefficients and the counting processes intensities are randomly generated.

where $\gamma_{i j k} \doteq \exp \left(\beta_{i j k}\right)-1$ is the relative size of the $i$ th stock jump upon any transition of the economy from state $j$ to state $k$.

In what follows, we will always assume that $S_{0}^{i}=1$, for all $i=0, \ldots, m$. The discounted stock prices $\tilde{S}_{t}^{i} \doteq S_{t}^{i} / S_{t}^{0}, i=0, \ldots, m$, are defined by

$$
\tilde{S}_{t}^{i}=\exp \left(\sum_{j=1}^{n}\left(\alpha_{i j}-r^{j}\right) \int_{0}^{t} I_{s}^{j} d s+\sum_{j=1}^{n} \sum_{k \in \mathcal{Y}_{j}} \beta_{i j k} N_{t}^{j k}\right),
$$

with dynamics

$$
d \tilde{S}_{t}^{i}=\tilde{S}_{t^{-}}^{i}\left(\sum_{j=1}^{n}\left(\alpha_{i j}-r^{j}\right) I_{t}^{j} d t+\sum_{j=1}^{n} \sum_{k \in \mathcal{Y}_{j}} \gamma_{i j k} d N_{t}^{j k}\right),
$$

$i=1, \ldots, m$. While, for $i=0$, we have $\tilde{S}_{t}^{0}=1$ and $d \tilde{S}_{t}^{0}=0$. 


\section{Arbitrage and Completeness}

In [25], the author provides sufficient conditions that assure the studied market to be arbitrage-free as well as complete. We will briefly recall latter result in what follows. For the sake of completeness, let us recall the notion of arbitrage, see, e.g., [13] for more details.

Definition 1. Let $\boldsymbol{\theta}=\left\{\boldsymbol{\theta}_{t}\right\}_{t \geq 0}$, be an $(m+1)$-dimensional stochastic process with

$$
\boldsymbol{\theta}_{t}=\left(\theta_{t}^{0}, \ldots, \theta_{t}^{m}\right),
$$

where $\theta_{t}^{i}$ represents the number of unit of the $i$ th asset held at time $t$. Assume that $\boldsymbol{\theta}$ is adapted to $\mathbf{F}^{Y}$ and that the shares of stocks, $\left(\theta_{t}^{1}, \ldots, \theta_{t}^{m}\right)$, are $\mathbf{F}^{Y}$ predictable. We call $\boldsymbol{\theta}$ a portfolio or an investment strategy.

Remark 2. We recall that any left-continuous, or deterministic, adapted process is predictable, see, e.g., [3, Sec. II.3].

The value process of a portfolio $\boldsymbol{\theta}$ at time $t$ is represented by

$$
V_{t}^{\theta} \doteq \sum_{i=0}^{m} \theta_{t}^{i} S_{t}^{i}
$$

while its discounted value process, at time $t$, is defined by

$$
\tilde{V}_{t}^{\theta} \doteq \sum_{i=0}^{m} \theta_{t}^{i} \tilde{S}_{t}^{i}
$$

Definition 3. The strategy $\boldsymbol{\theta}$ is said to be self-financing if

$$
d V_{t}^{\theta}=\sum_{i=0}^{m} \theta_{t}^{i} d S_{t}^{i}
$$

namely if, recalling that $d \tilde{S}_{t}^{0}=0$, it holds

$$
d \tilde{V}_{t}^{\theta}=\sum_{i=1}^{m} \theta_{t}^{i} d \tilde{S}_{t}^{i}
$$

Let $\tilde{\boldsymbol{\Lambda}}=\left(\tilde{\lambda}_{j k}\right)_{j, k \in \mathcal{Y}}$ be an infinitesimal matrix equivalent to $\boldsymbol{\Lambda}$ in the sense that $\tilde{\lambda}_{j k}=0$ if and only if $\lambda_{j k}=0$, then, thanks to the Girsanov theorem for multivariate counting processes, see, e.g., [15, Sec. VI.2], there exists a measure 
$\tilde{\mathbb{P}}$, equivalent to $\mathbb{P}$, under which $Y$ is a Markov chain with infinitesimal matrix $\tilde{\Lambda}$. Thus, the process $\left\{\tilde{M}_{t}^{j k}\right\}_{t \geq 0}, j \in \mathcal{Y}, k \in \mathcal{Y}_{j}$, defined by

$$
d \tilde{M}_{t}^{j k} \doteq d N_{t}^{j k}-I_{t}^{j} \tilde{\lambda}_{j k} d t
$$

and $\tilde{M}_{0}^{j k}=0$, are zero mean, mutually orthogonal martingales with respect to $\left(\mathbf{F}^{Y}, \tilde{\mathbb{P}}\right)$., therefore we can rewrite the discounted stock price dynamics $(3)$, as follows

$$
d \tilde{S}_{t}^{i}=\tilde{S}_{t^{-}}^{i}\left(\sum_{j=1}^{n}\left(\alpha_{i j}-r^{j}+\sum_{k \in \mathcal{Y}_{j}} \gamma_{i j k} \tilde{\lambda}_{j k}\right) I_{t}^{j} d t+\sum_{j=1}^{n} \sum_{k \in \mathcal{Y}_{j}} \gamma_{i j k} d \tilde{M}_{t}^{j k}\right)
$$

$i=1, \ldots, m$.

Definition 4. An arbitrage is a self-financing portfolio value process $\left\{V_{t}\right\}_{t \geq 0}$ such that $V_{0}=0$ and, for some time $T>0$,

$$
\mathbb{P}\left(V_{T} \geq 0\right)=1, \quad \mathbb{P}\left(V_{T}>0\right)>0 .
$$

By Def. 4, it follows that, in a given financial market, we have arbitrage opportunities, if we can trade starting with zero capital at time $t=0$, being sure to have at some later, but finite, time $T>0$, no money loss, moreover with a positive probability of gaining it.

Definition 5. A probability measure $\tilde{\mathbb{P}}$ is said to be a risk-neutral measure if

1. $\tilde{\mathbb{P}}$ and $\mathbb{P}$ are equivalent,

2. under $\tilde{\mathbb{P}}$, the discounted stock price process $\left\{\tilde{S}_{t}^{i}\right\}_{t \geq 0}$ is a martingale for every $i=1, \ldots, m$.

Theorem 6 (First fundamental theorem of asset pricing). If a market model has a risk-neutral probability measure, then it does not admit aribtrage.

Proof. See, e.g., [28, Sec. 5.4].

In order to see if the market model we are considering is arbitrage-free, let us observe that point (i) of Def. 5 is satisfied by $\tilde{\mathbb{P}}$. Moreover, we know that the discounted stock price processes are martingales under $\tilde{\mathbb{P}}$ if the drift term in eq. (5) vanishes, namely if

$$
\alpha_{i j}-r^{j}+\sum_{k \in \mathcal{Y}_{j}} \gamma_{i j k} \tilde{\lambda}_{j k}=0
$$


$j=1, \ldots, n, i=1, \ldots, m$. Thus, the existence of a risk-neutral measure is equivalent to the existence of a solution $\tilde{\lambda}_{j}$ to (6) with all positive entries. We summarize latter result in the following statement.

Proposition 7. A CTMC model is arbitrage-free if there exists an infinitesimal matrix $\tilde{\boldsymbol{\Lambda}}=\left(\tilde{\lambda}_{j k}\right)_{j, k \in \mathcal{Y}}$, equivalent to $\boldsymbol{\Lambda}$, such that its components are nonnegative and satisfy equations (6).

Moreover, we have the following result

Theorem 8. Let $\tilde{\mathbb{P}}$ be a risk-neutral measure, and let $\left\{V_{t}^{\theta}\right\}_{t \geq 0}$ be the value of a self-financing portfolio $\boldsymbol{\theta}$. Under $\tilde{\mathbb{P}}$, the discounted portfolio value $\left\{\tilde{V}_{t}^{\theta}\right\}_{t \geq 0}$ is a martingale, namely, for any $0 \leq s<t$, we have

$$
\tilde{V}_{s}^{\theta}=\mathbb{E}\left[\tilde{V}_{t}^{\theta} \mid \mathcal{F}_{s}\right]
$$

Proof. See, e.g., [25, Sec. 2.D].

In force of the previous results, in what follows we shall assume that the market is arbitrage-free. In order to analyse the completeness of the CTMC model introduced before, let us introduce the concept of T-claim, that is a contractual payment due at finite time $T>0$.

Definition 9. A contingent claim with date of maturity (or exercise date) $0<T<+\infty$, also called a $T$-claim, is any $\mathcal{F}_{T}^{Y}$-measurable random variable $H$ with finite expected value.

A $T$-claim is called simple if it is of the form $H=\phi\left(Y_{T}, S_{T}^{1}, \ldots, S_{T}^{m}\right)$, the function $\phi$ being defined as the contract function. The interpretation of Def. 9 is that a $T$-claim is a contract which guarantees the holder to obtain a payoff of $H$, which can be positive or negative, at maturity $T$.

Definition 10. We say that a $T$-claim $H$ can be replicated, or that it is hedgeable, if there exists a self-financing portfolio $\boldsymbol{\theta}$ such that the following holds true

$$
\tilde{V}_{T}^{\theta}=\tilde{H}, \quad \tilde{\mathbb{P}}_{\text {-a.s. } .}
$$

Proposition 11. Let $H$ be a $T$-claim, then $H$ is hedgeable if and only if there exists a predictable processes $\left\{\xi_{t}^{i}\right\}_{t \geq 0}, i=1, \ldots, m$, such that

$$
\sum_{i=1}^{m} \xi_{t}^{i} \tilde{S}_{t^{-}}^{i} \gamma_{i j k}=\zeta_{t}^{j k}, \quad \text { for all } j \in \mathcal{Y}, k \in \mathcal{Y}_{j}
$$


where $\left\{\zeta_{t}^{j k}\right\}_{t \geq 0}$ are $\mathbf{F}^{Y}$-predictable and integrable processes, such that

$$
\tilde{H}=\tilde{\mathbb{E}}[\tilde{H}]+\int_{0}^{T} \sum_{j=1}^{n} \sum_{k \in \mathcal{Y}_{j}} \zeta_{t}^{j k} d \tilde{M}_{t}^{j k}
$$

Proof. See [25, Sec. 2.F].

Notice that any $T$-claim $H$, as defined in Definition 9, can be represented as in equation (9), see, e.g., [7, Sec. 1.3]. Conversely, any random variable of the form (9) is a $T$-claim.

Definition 12. A market model is called complete if every $T$-claim is hedgeable.

Theorem 13 (Second fundamental theorem of asset pricing). A market model is complete if and only if it admits a unique risk-neutral measure.

Proof. See, e.g., [28, Sec. 5.4].

Exploiting Th. 13 and the conditions stated in (8), we can derive the following result for our CTMC market model.

Proposition 14. The Markov chain market is complete if $\operatorname{rank}\left(\boldsymbol{\Gamma}_{j}\right)=n_{j}$, which can be fulfilled for each $j=1, \ldots, n$, only if

$$
m \geq \max _{j \in \mathcal{Y}} n_{j}
$$

where $n_{j}$ is the number of the states that are directly accessible from state $j \in \mathcal{Y}$, and $\boldsymbol{\Gamma}_{j} \doteq\left(\gamma_{i j k}\right)_{i=1, \ldots, m}^{k \in \mathcal{Y}_{j}}$.

Proof. See, e.g., [25, Sec. 2.F].

\section{Market Models Examples}

The Markov chain model introduced in Sec. 2 is extremely versatile. Thanks to the great number of parameters and variables it depends on, and taking into account the properties characterizing a general counting processes, we have that such a model shares various interesting financial properties. Moreover, we underline that a lot of market model can be derived from the CTMC model by suitably choosing the parameters and the processes distribution characterising the CTMC model itself, as described in what follows where, for the sake of 
simplicity and without loss of generality, we will consider just one risky asset. In particular, we rewrite eq. (2), as follows

$$
S_{t}=S_{0} \exp \left(\sum_{j=1}^{n} \alpha_{j} \int_{0}^{t} I_{s}^{j} d s+\sum_{j=1}^{n} \sum_{k \in \mathcal{Y}_{j}} \beta_{j k} N_{t}^{j k}\right),
$$

where the parameters $\alpha_{j}$ and $\beta_{j k}$ play the same roles as the $\alpha_{i j}$ and $\beta_{i j k}$ in eq. (2), and analogously for the $\gamma_{i j k}$, etc. The dynamics described in eq. (10), reads as follow

$$
d S_{t}=S_{t} \sum_{j=1}^{n} \alpha_{j} I_{t}^{j} d t+S_{t^{-}} \sum_{j=1}^{n} \sum_{k \in \mathcal{Y}_{j}} \gamma_{j k} d N_{t}^{j k}
$$

Furthermore, we assume that the rate of interest is not state-wise dependent, namely $r=r^{j}$, for all $j \in \mathcal{Y}$, thus the bond price process (1), which we refer to as $B_{t}$ instead of $S_{t}^{0}$, is given by

$$
B_{t}=B_{0} e^{r t}
$$

\subsection{The Geometric Poisson Model}

We consider a stock modeled as a geometric Poisson process, therefore the asset evolution in time reads as follow

$$
S_{t}=S_{0} \exp \left((\alpha-\lambda \sigma) t+\log (1+\sigma) N_{t}\right),
$$

where $\alpha$ and $\sigma$ are assumed to be constant, $\sigma>-1, \sigma \neq 0$, and $\left\{N_{t}\right\}_{t \geq 0}$ is a Poisson process with intensity $\lambda>0$ under the so-called real world probability measure $\mathbb{P}$. From eq. (12), we have

$$
d S_{t}=(\alpha-\lambda \sigma) S_{t} d t+\sigma S_{t^{-}} d N_{t}
$$

In order to replicate the dynamic described by eq. (12) by mean of the CTMC model, we set $n=2$, and then $\mathcal{Y}=\{1,2\}$, and we consider $\alpha_{1}=\alpha_{2}=$ $\alpha-\lambda \sigma$, which implies that

$$
\sum_{j=1}^{2} \alpha_{j} \int_{0}^{t} I_{u}^{j} d u=(\alpha-\lambda \sigma) \sum_{j=1}^{2} \int_{0}^{t} I_{u}^{j} d u=(\alpha-\lambda \sigma) t
$$

Moreover, we set $\beta_{12}=\beta_{21}=\log (\sigma+1)$ and $\lambda_{12}=\lambda_{21}=\lambda$, defining the process $\left\{N_{t}\right\}_{t \geq 0}$ as follows

$$
N_{t} \doteq N_{t}^{12}+N_{t}^{21},
$$


which then turns to be a Poisson process with intensity $\lambda$. Therefore, we have

$$
\beta_{12} N_{t}^{12}+\beta_{21} N_{t}^{21}=\log (\sigma+1) N_{t},
$$

which allows us to obtain eq. (12). Notice that, for the geometric Poisson process, the no arbitrage condition stated in eq. (6) reduces to

$$
\tilde{\lambda}=\lambda+\frac{r-\alpha}{\sigma}>0
$$

\subsection{The Geometric Brownian Motion Model}

Let us assume that both $\alpha$ and $\sigma>0$, namely the drift and the volatility of the Black-Scholes model, are constants. Then, the stock price process $S_{t}$ satisfies the following equation

$$
S_{t}=S_{0} \exp \left(\left(\alpha-\frac{1}{2} \sigma^{2}\right) t+\sigma W_{t}\right),
$$

where $\left\{W_{t}\right\}_{t \geq 0}$ is a standard Brownian motion.

Therefore, exploiting the Itô-Doeblin formula, we obtain that the stock dynamics reads as follows

$$
d S_{t}=\alpha S_{t} d t+\sigma S_{t} d W_{t}
$$

a sample trajectory of which is reported in Figure 2, where the mean function, shown in red, is given by

$$
\begin{aligned}
\mathbb{E}\left[S_{t}\right] & =S_{0} \exp \left(\alpha t-\frac{1}{2} \sigma^{2} t\right) \mathbb{E}\left[e^{\sigma W_{t}}\right] \\
& =S_{0} \exp (\alpha t)
\end{aligned}
$$

since $\sigma W_{t} \sim \mathcal{N}\left(0, \sigma^{2} t\right)$, and thanks to the moment-generating formula for a normal distribution.

In order to replicate the aforementioned Black-Scholes model in the CTMC model setting, we use the following result.

Proposition 15. Let $\left\{N_{t}\right\}_{t \geq 0}$ be a Poisson process with intensity $\lambda>0$, and let $W=\left\{W_{t}\right\}_{t \geq 0}$ be a standard Brownian motion. Consider the process $\left\{C_{t}\right\}_{t \geq 0}$ given by

$$
C_{t} \doteq \frac{N_{t}-t \lambda}{\sqrt{\lambda}} .
$$

Then, for any fixed $t \geq 0$, the random variable $C_{t}$ converges weakly to $W_{t}$ as $\lambda \rightarrow+\infty$. 


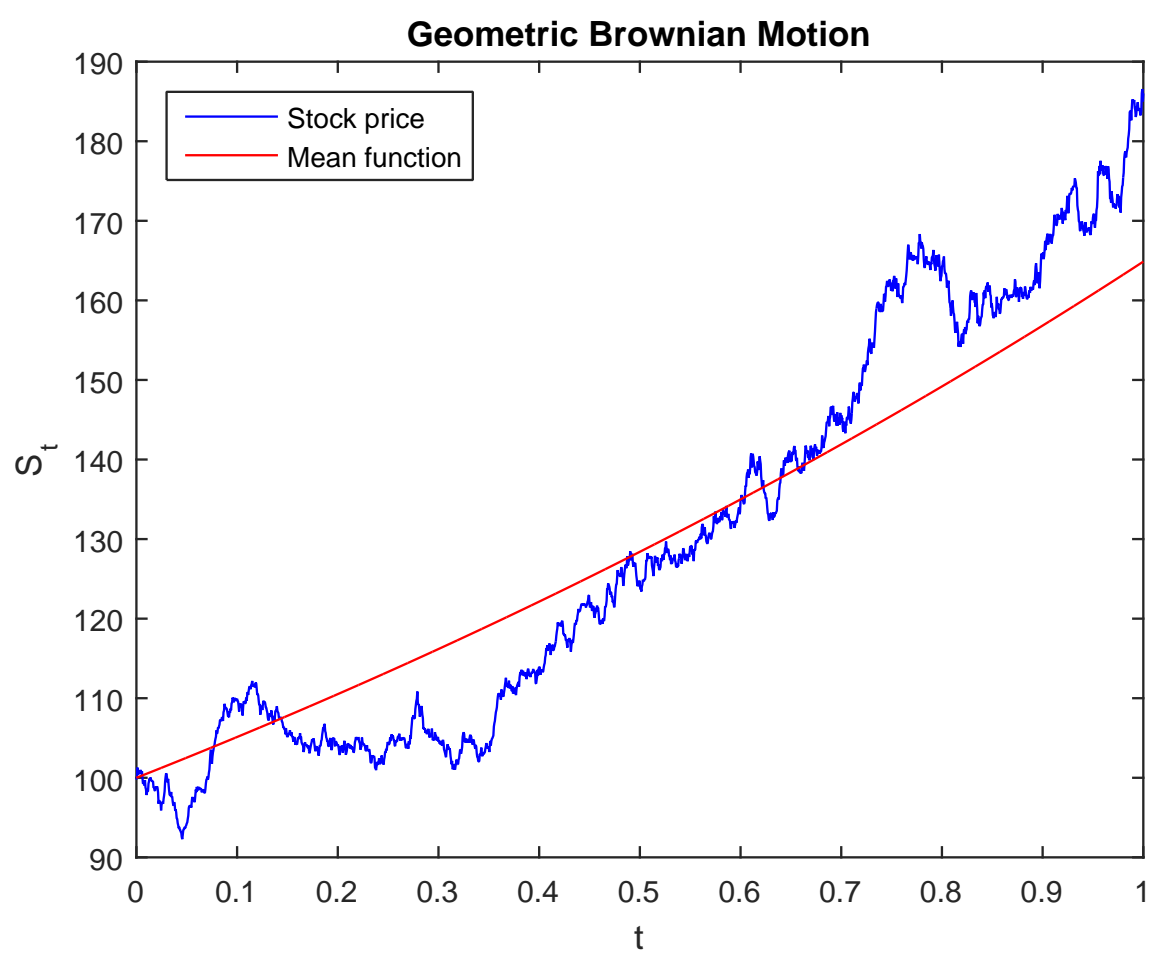

Figure 2: Trajectory of a geometric Brownian motion (13), with $S_{0}=$ 100, $T=1, \alpha=0.5$ and $\sigma=0.2$.

Proof. Thanks to the Central Limit Theorem, if $N_{i} \sim \operatorname{Po}(\mu)$ for $i=1,2, \ldots$ is a sequence of independent and identically distributed random variables, then obviously we have $N_{1}+\ldots+N_{n} \sim \operatorname{Po}(n \mu)$, and the random variable

$$
\frac{N_{1}+\ldots+N_{n}-n \mu}{\sqrt{n \mu}}
$$

converges weakly, as $n \rightarrow+\infty$, to a normally distributed random variable with zero mean and variance 1 . Furthermore, if $n \rightarrow+\infty$, then $\lambda \doteq n \mu \rightarrow+\infty$. In the considered case, $N_{t} \sim \operatorname{Po}(t \lambda)$, where we can consider $\lambda \doteq n \mu$. Thus

$$
\frac{N_{t}-t \lambda}{\sqrt{t \lambda}}
$$

converges weakly to a $\mathcal{N}(0,1)$ random variable as $\lambda \rightarrow+\infty$. Thanks to the 
properties of the normal distribution we have that the random variable

$$
\frac{N_{t}-t \lambda}{\sqrt{\lambda}}
$$

converges weakly to a $\mathcal{N}(0, t)$ distribution as $\lambda \rightarrow+\infty$.

In particular, we have that the process $\left\{C_{t}\right\}_{t \in[0, T]}$ converges in distribution to $\left\{W_{t}\right\}_{t \in[0, T]}$, with the notions of convergence on the space of right-continuous with left limits, càdlàg, functions, see, e.g., [20].

We set the counting processes and parameters, as follows:

$$
\begin{array}{lr}
\lambda_{j k}=\lambda, & \text { for all } j \in \mathcal{Y}, k \in \mathcal{Y}_{j}, \\
\alpha_{j}=\alpha-\frac{1}{2} \sigma^{2}-\sigma \sqrt{\lambda}, & \text { for all } j \in \mathcal{Y}, \\
\beta_{j k}=\frac{\sigma}{\sqrt{\lambda}}, & \text { for all } j \in \mathcal{Y}, k \in \mathcal{Y}_{j},
\end{array}
$$

with $\lambda>0$. The process $\left\{N_{t}\right\}_{t \geq 0}$ is a Poisson process with density $\lambda$ defined as

$$
N_{t} \doteq \sum_{j=1}^{n} \sum_{k \in \mathcal{Y}_{j}} N_{t}^{j k}
$$

then we are left with

$$
S_{t}=S_{0} \exp \left(\left(\alpha-\frac{1}{2} \sigma^{2}\right) t+\sigma \frac{N_{t}-\lambda t}{\sqrt{\lambda}}\right),
$$

and so it converges weakly to eq. (13), as $\lambda \rightarrow+\infty$, thanks to Prop. 15. A sample path example for the process (16) is shown in Figure 3, where the mean function of $S_{t}$ is given by

$$
\begin{aligned}
\mathbb{E}\left[S_{t}\right] & =S_{0} \exp \left(\alpha t-\frac{t \sigma^{2}}{2}-\sigma \sqrt{\lambda}\right) \mathbb{E}\left[e^{\frac{\sigma}{\sqrt{\lambda}} N_{t}}\right] \\
& =S_{0} \exp \left(\alpha t-\frac{t \sigma^{2}}{2}-\sigma \sqrt{\lambda}+t \lambda\left(e^{\frac{\sigma}{\sqrt{\lambda}}}-1\right)\right),
\end{aligned}
$$

since $N_{t} \sim \operatorname{Po}(t \lambda)$ and thanks to the moment-generating formula for a Poisson distribution.

Remark 16. We would like to underline that the mean function (17) of the price process (16) tends to the mean function (14) of the geometric Brownian motion as $\lambda \rightarrow+\infty$. 


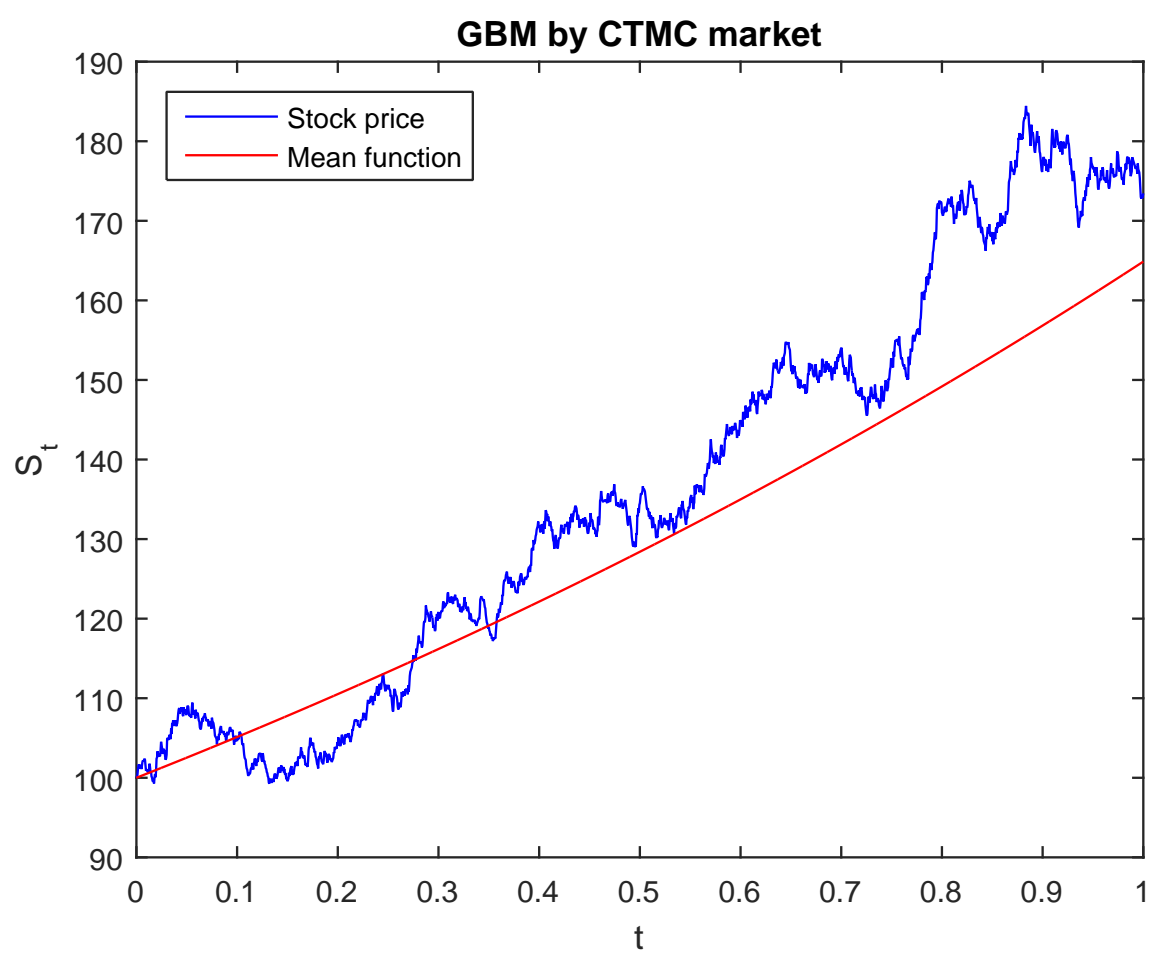

Figure 3: Trajectory of an asset price process driven by (16), with $S_{0}=100, T=1, \alpha=0.5, \sigma=0.2$ and $\lambda=5000$.

In particular, the following result holds true.

Proposition 17. Let $\sigma>0, S_{0}>0$ and $\alpha$ be fixed constants. Let $\left\{X_{t}\right\}_{t \geq 0}$ be a stochastic process given by

$$
X_{t}=S_{0} \exp \left(\left(\alpha-\frac{1}{2} \sigma^{2}\right) t+\sigma \frac{N_{t}-\lambda t}{\sqrt{\lambda}}\right),
$$

where $\left\{N_{t}\right\}_{t \geq 0}$ is a Poisson process with intensity $\lambda>0$. Let $\left\{S_{t}\right\}_{t \geq 0}$ be a stochastic process given by

$$
S_{t}=S_{0} \exp \left(\left(\alpha-\frac{1}{2} \sigma^{2}\right) t+\sigma W_{t}\right),
$$

where $\left\{W_{t}\right\}_{t \geq 0}$ is a standard Brownian motion. Thus, for any $t \geq 0, X_{t} \stackrel{d}{\rightarrow} S_{t}$ as $\lambda \rightarrow+\infty$. 


\subsection{The Brownian Motion with a Compound Poisson Component Model}

Let us recall, for the sake of completeness, the definition of compound Poisson process, along with a result that we will exploit later on.

Definition 18. Let $\left\{N_{t}\right\}_{t \geq 0}$ be a Poisson process with intensity $\lambda$, and let $\left\{Y_{i}, i \in \mathbb{N}^{+}\right\}$, be a sequence of independent and identically distributed random variables, with mean $\beta=\mathbb{E}\left[Y_{i}\right]$, also independent of the Poisson process $\left\{N_{t}\right\}_{t \geq 0}$. Then the process $\left\{Q_{t}\right\}_{t \geq 0}$ defined by

$$
Q_{t} \doteq \sum_{i=1}^{N_{t}} Y_{i}, \quad t \geq 0
$$

is called a compound Poisson process.

Theorem 19 (Decomposition of a compound Poisson process). Let $y_{1}, \ldots$, $y_{m}$ be a finite set of nonzero numbers and let $p\left(y_{1}\right), \ldots, p\left(y_{m}\right)$ be positive numbers that sum to 1 . Let $\lambda>0$ be given, and let $\left\{N_{t}^{1}\right\}_{t \geq 0}, \ldots,\left\{N_{t}^{m}\right\}_{t \geq 0}$ be independent Poisson processes, each $N_{t}^{i}$ having intensity $\lambda p\left(y_{i}\right)$ for all $i=1, \ldots, m$. Define

$$
Q_{t} \doteq \sum_{i=1}^{m} y_{i} N_{t}^{i}, \quad t \geq 0
$$

Then $\left\{Q_{t}\right\}_{t \geq 0}$ is a compound Poisson process. In particular, if $Y_{i}$ is the size of the $i$-th jump, $i=1, \ldots, m$, and

$$
N_{t} \doteq \sum_{i=1}^{m} N_{t}^{i}, \quad t \geq 0,
$$

is the total number of jumps on the time interval $[0, t)$, then $\left\{N_{t}\right\}_{t \geq 0}$ is a Poisson process with intensity $\lambda$, the random variables $Y_{1}, Y_{2}, \ldots$ are independent with $\mathbb{P}\left(Y_{k}=y_{i}\right)=p\left(y_{i}\right)$ for $i=1, \ldots, m$, the random variables $Y_{1}, \ldots, Y_{m}$ are independent of $N_{t}$, and

$$
Q_{t} \doteq \sum_{k=1}^{N_{t}} Y_{k}, \quad t \geq 0
$$

Proof. See, e.g., [28, Sec. 11.3].

Let $(\Omega, \mathcal{F}, \mathbb{P})$ be a probability space on which is defined a (standard) Brownian motion $W=\left\{W_{t}\right\}_{t \in[0, T]}$ and a compound Poisson process $Q=\left\{Q_{t}\right\}_{t \in[0, T]}$ 
with underlying Poisson process $N=\left\{N_{t}\right\}_{t \geq 0}$ having intensity $\lambda>0$ and jump sizes given by a sequence $A_{1}, A_{2}, \ldots$ of independent and identically distributed random variables, each of which is independent of $N$, and with $\beta \doteq \mathbb{E}\left[A_{i}\right]$, see Def. 18. Suppose that the random variables $A_{1}, A_{2}, \ldots$ can take a finite number of value $a_{1}, \ldots, a_{c}$, such that $-1<a_{1}<\ldots<a_{c}$ and $a_{\ell} \neq 0$ for all $\ell=1, \ldots, c$, with probability $p\left(a_{1}\right), \ldots, p\left(a_{c}\right)$. Let $\left\{\mathcal{F}_{t}\right\}_{t \in[0, T]}$ be the filtration jointly generated by the Brownian motion and the compound Poisson process. Taking into account previous definitions, let us model the price process by the following stochastic differential equation

$$
\begin{aligned}
d S_{t} & =\alpha S_{t} d t+\sigma S_{t} d W_{t}+S_{t^{-}} d\left(Q_{t}-\beta \lambda t\right) \\
& =(\alpha-\beta \lambda) S_{t} d t+\sigma S_{t} d W_{t}+S_{t^{-}} d Q_{t} .
\end{aligned}
$$

Then we have the following result

Theorem 20. The solution to (18) is

$$
\begin{aligned}
S_{t} & =S_{0} \exp \left(\left(\alpha-\beta \lambda-\frac{1}{2} \sigma^{2}\right) t+\sigma W_{t}\right) \prod_{i=1}^{N_{t}}\left(A_{i}+1\right) \\
& =S_{0} \exp \left(\left(\alpha-\beta \lambda-\frac{1}{2} \sigma^{2}\right) t+\sigma W_{t}+\sum_{i=1}^{N_{t}} \log \left(A_{i}+1\right)\right) .
\end{aligned}
$$

Proof. See, e.g., [28, Sec. 11.7].

A sample path of an asset price having dynamic given by eq. (19) can be seen in Figure 4.

In order to get eq. (18) from the corresponding eq. (11), in the case of $c+1$ Markov chains, let us consider $c+1$ independent $n$-dimensional homogeneous continuous-time Markov chains $\left\{Y_{t}^{\ell}\right\}_{t \geq 0}, \ell=0, \ldots, c$. Then we will combine the results stated in Sec. 4.2 together with Th. 19.

In the case of $c$ independent homogeneous continuous-time Markov chain, the stock price process eq. (10) reads as follow

$$
S_{t}=S_{0} \exp \left(\sum_{\ell=0}^{c} \sum_{j=1}^{n} \alpha_{\ell j} t+\sum_{\ell=0}^{c} \sum_{j=1}^{n} \sum_{k \in \mathcal{Y}_{j}} \beta_{\ell j k} N_{t}^{\ell j k}\right),
$$

where the new index, $\ell$, denote that the coefficient is due to the $\ell$ th matrix. We start making the following assumptions for $\ell=0, \ldots, c$ :

$$
\alpha_{\ell j}=\alpha_{\ell} \quad \text { for all } j \in \mathcal{Y},
$$




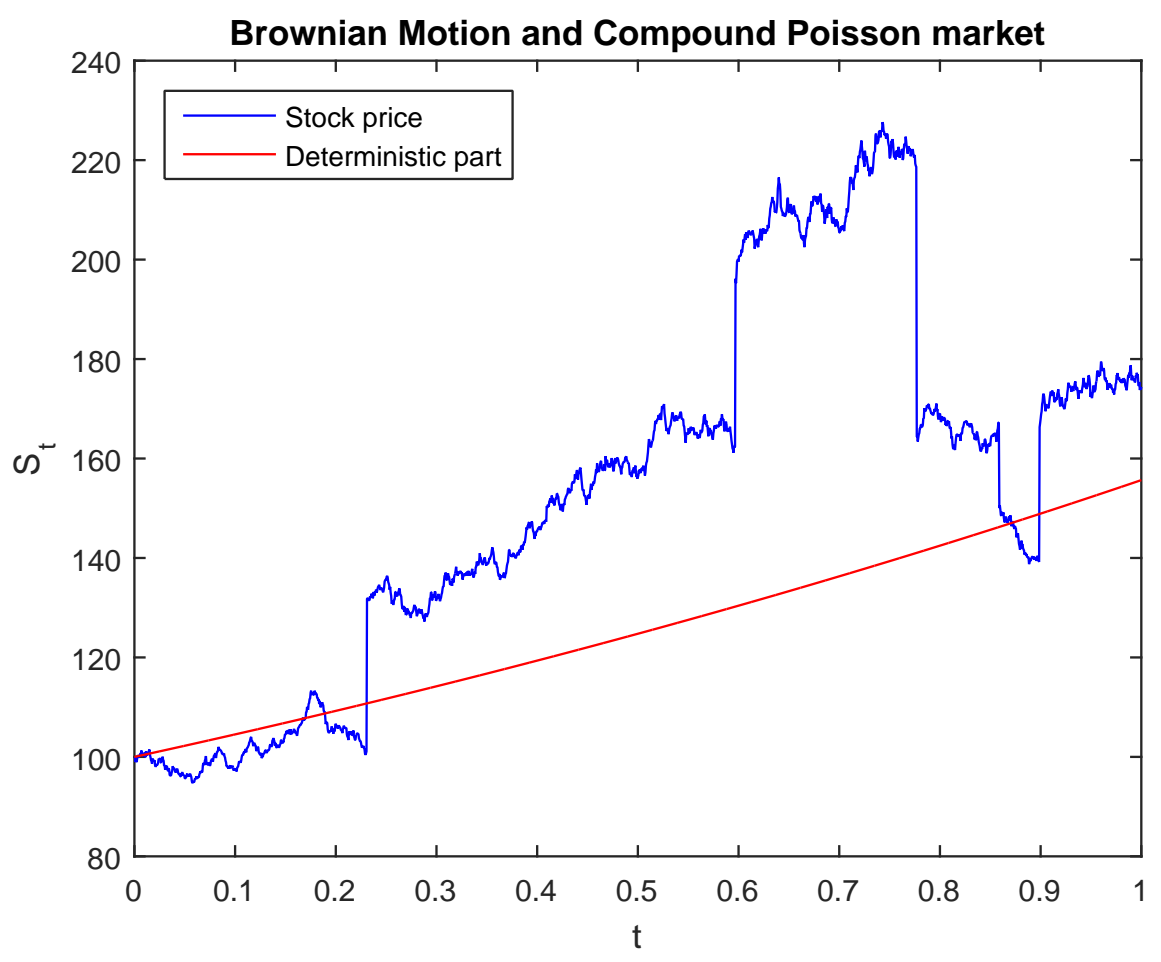

Figure 4: Trajectory of a geometric Brownian motion and compound Poisson process asset price (19), with $S_{0}=100, T=1, \lambda=5.5, \alpha=0.4$ and $\sigma=0.2$.

$$
\begin{aligned}
& \beta_{\ell j k}=\beta_{\ell} \\
& \text { for all } j \in \mathcal{Y}, k \in \mathcal{Y}_{j} \text {, } \\
& \lambda_{\ell j k}=\lambda_{\ell} \\
& \text { for all } j \in \mathcal{Y}, k \in \mathcal{Y}_{j} \text {. }
\end{aligned}
$$

Notice that the second assumption implies that $\gamma_{\ell j k}=\gamma_{\ell}$ for all $j \in \mathcal{Y}, k \in \mathcal{Y}_{j}$. We then assume that $N_{t}^{\ell} \doteq \sum_{j=1}^{n} \sum_{k \in \mathcal{Y}_{j}} N_{t}^{\ell j k}$ is a Poisson process with intensity $\lambda_{\ell}$ for all $\ell=0, \ldots, c$, so that eq. (20) becomes

$$
S_{t}=S_{0} \exp \left(\sum_{\ell=0}^{c} \alpha_{\ell} t+\sum_{\ell=0}^{c} \beta_{\ell} N_{t}^{\ell}\right)
$$

In what follows we are going to use the Markov chain $\ell=0$ to model the Brownian motion part, while the remaining $c$ Markov chains, will be used to model the compound Poisson process part. In particular, for the first part, we 


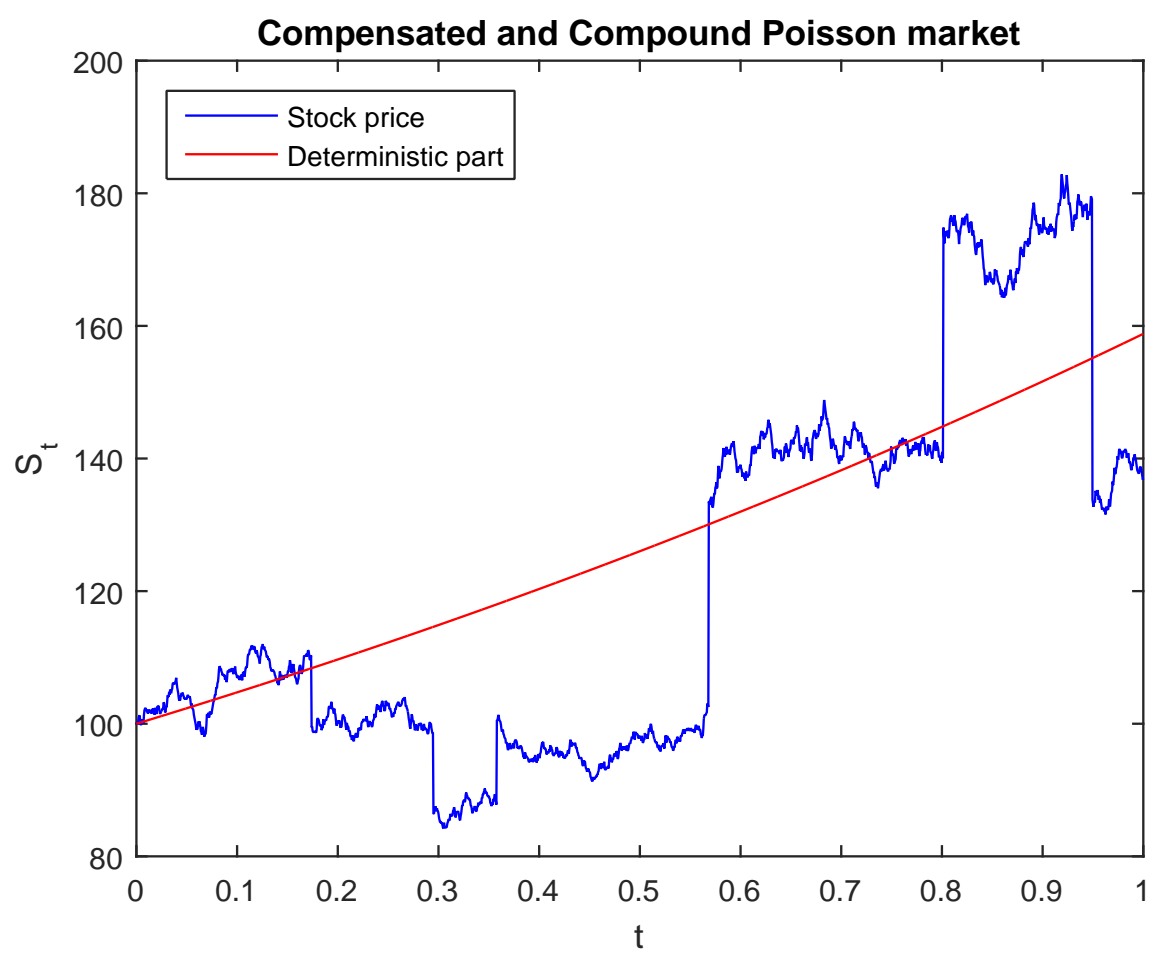

Figure 5: Trajectory of an asset price process driven by (23), with $S_{0}=100, T=1, \lambda=5.5, \alpha=0.4, \sigma=0.2$ and $\lambda_{0}=5000$.

follow Sec. 4.2, considering $\alpha_{0}=\alpha-\frac{1}{2} \sigma^{2}-\sigma \sqrt{\lambda_{0}}$ and $\beta_{0}=\frac{\sigma}{\sqrt{\lambda}}$, which implies the following new form for eq. (21)

$$
\begin{array}{ll}
S_{t}=S_{0} \exp \left(\left(\alpha-\frac{1}{2} \sigma^{2}\right) t+\sigma \frac{N_{t}^{0}-\lambda_{0} t}{\sqrt{\lambda_{0}}}\right. & \\
& \left.+\sum_{\ell=1}^{c} \alpha_{\ell} t+\sum_{\ell=1}^{c} \beta_{\ell} N_{t}^{\ell}\right) .
\end{array}
$$

In order to obtain the compound Poisson process $Q$, we use the decomposition of compound Poisson processes as stated by Th. 19. In particular, we assume that $\gamma_{\ell}=a_{\ell}$, for every $\ell=1, \ldots, c$, and that $\lambda_{\ell}, \ell=1, \ldots, c$ take values such that

$$
\lambda_{\ell} \doteq \lambda p\left(\gamma_{\ell}\right)=\lambda \mathbb{P}\left(A_{i}=\gamma_{\ell}\right)
$$


which implies that

$$
\lambda=\sum_{\ell=1}^{c} \lambda_{\ell}
$$

therefore

$$
Q_{t}=\sum_{\ell=1}^{c} \gamma_{\ell} N_{t}^{\ell}, \quad 0 \leq t \leq T,
$$

which is nothing but the alternative definition of the compound Poisson process $Q$.

Finally, we set $\alpha_{\ell}=-\gamma_{\ell} \lambda_{\ell}$ for all $\ell=1, \ldots, c$, so that we have

$$
\begin{aligned}
\sum_{\ell=1}^{c} \alpha_{\ell}=-\sum_{\ell=1}^{c} \gamma_{\ell} \lambda_{\ell}=-\lambda \sum_{\ell=1}^{c} \frac{\gamma_{\ell} \lambda_{\ell}}{\lambda} & \\
& =-\lambda \sum_{\ell=1}^{c} \gamma_{\ell} p\left(\gamma_{\ell}\right)=-\lambda \mathbb{E}\left[A_{i}\right]=-\lambda \beta
\end{aligned}
$$

Therefore, recalling that $\gamma_{\ell}=e^{\beta_{\ell}}-1$ and consequently $\beta_{\ell}=\log \left(\gamma_{\ell}+1\right)$, eq. (22) reads as follow

$$
\begin{aligned}
S_{t}=S_{0} \exp \left(\left(\alpha-\frac{1}{2} \sigma^{2}-\lambda \beta\right) t\right. & \\
& \left.+\sigma \frac{N_{t}^{0}-\lambda_{0} t}{\sqrt{\lambda_{0}}}+\sum_{\ell=1}^{c} \log \left(\gamma_{\ell}+1\right) N_{t}^{\ell}\right),
\end{aligned}
$$

and, exploiting Th. 19 together with Prop. 15, we have that $S_{t}$ weakly converges to eq. (19) as $\lambda_{0}$ goes to $+\infty$. In Figure 5 we report an example of an asset price trajectory as stated by eq. (23).

For the sake of clarity, we summarize the results obtained so far in the following proposition.

Proposition 21. Let $\alpha, \beta$ and $\sigma>0$ be fixed constants. Let $\left\{X_{t}\right\}_{t \geq 0}$ be a stochastic process given by

$$
X_{t}=S_{0} \exp \left(\left(\alpha-\frac{1}{2} \sigma^{2}-\lambda \beta\right) t+\sigma \frac{N_{t}^{0}-\lambda_{0} t}{\sqrt{\lambda_{0}}}+\sum_{\ell=1}^{c} \log \left(\gamma_{\ell}+1\right) N_{t}^{\ell}\right)
$$

where $N=\left\{N_{t}^{\ell}\right\}_{t \geq 0}$ is a Poisson process with intensity $\lambda_{\ell}>0, \ell=0, \ldots, c$, and $\gamma_{1}, \ldots, \gamma_{c}$, are constants such that $-1<\gamma_{1}<\ldots<\gamma_{c}$ and $\gamma_{\ell} \neq 0, \ell=1, \ldots, c$. 
Let $\left\{S_{t}\right\}_{t \geq 0}$ be a stochastic process given by

$$
S_{t}=S_{0} \exp \left(\left(\alpha-\beta \lambda-\frac{1}{2} \sigma^{2}\right) t+\sigma W_{t}+\sum_{i=1}^{N_{t}} \log \left(\Gamma_{i}+1\right)\right),
$$

where $\left\{W_{t}\right\}_{t \geq 0}$ is a standard Brownian motion, while $\Gamma_{1}, \Gamma_{2}, \ldots$, are independent and identically distributed random variables, each of which is independent of $N$, taking finitely many values $\gamma_{\ell}$ with probability $\lambda_{\ell} / \lambda, \ell=1, \ldots, c$, and such that $\beta=\mathbb{E}\left[\Gamma_{i}\right]$. Then, for any fixed $t \geq 0, X_{t} \stackrel{d}{\rightarrow} S_{t}$ as $\lambda_{0} \rightarrow+\infty$.

\section{Weak Convergence Analysis}

In this section we want to test the weak convergences rigorously analysed in Sec. 4 from a qualitative point of view. In particular, we simulate the process (16) for different values of $\lambda$ and of $\sigma$, which are the parameters that sets the jump intensities and sizes, and, in Figures 6 and 7, we report the graphs associated to such numerical simulations. We would like to underline that the following numerical results have been obtained without a particular care of the computational efficiency of the implemented algorithms. The latter has implied rather long time of computations which, as an example, can be easily overcome by using ad hoc techniques, as the ones developed, e.g., in $[9,8]$ and references therein, namely using the so called chaos decomposition approach.

We have used the same code as the one exploited for Figure 3, but taking into account various values of $\lambda$ and $\sigma$. In particular, we have considered the values $\{10,50,200,2000\}$ for the jump intensities, resp. the values $\{0.05,0.3,1.25\}$, for the parameter $\sigma$. As in Figure 3, the red line represents the mean function of $\left\{S_{t}\right\}_{t}$, while the blue line is the price itself.

Considering the obtained plots, we can deduce, according to Prop. 17, that the more $\lambda$ increases, the more the trajectory of a process given by eq. (16) is similar to the one of a geometric Brownian motion.

Regarding $\sigma$, one can easily see that the more its value increases, the more the trajectory of the price process tends to be distant from its mean function.

Recalling that the mean function of the process, defined by equation (16), is represented as in equation (17), namely

$$
\mathbb{E}\left[X_{t}\right]=S_{0} \exp \left(\alpha t-\frac{t \sigma^{2}}{2}-t \sigma \sqrt{\lambda}+t \lambda\left(e^{\frac{\sigma}{\sqrt{\lambda}}}-1\right)\right),
$$



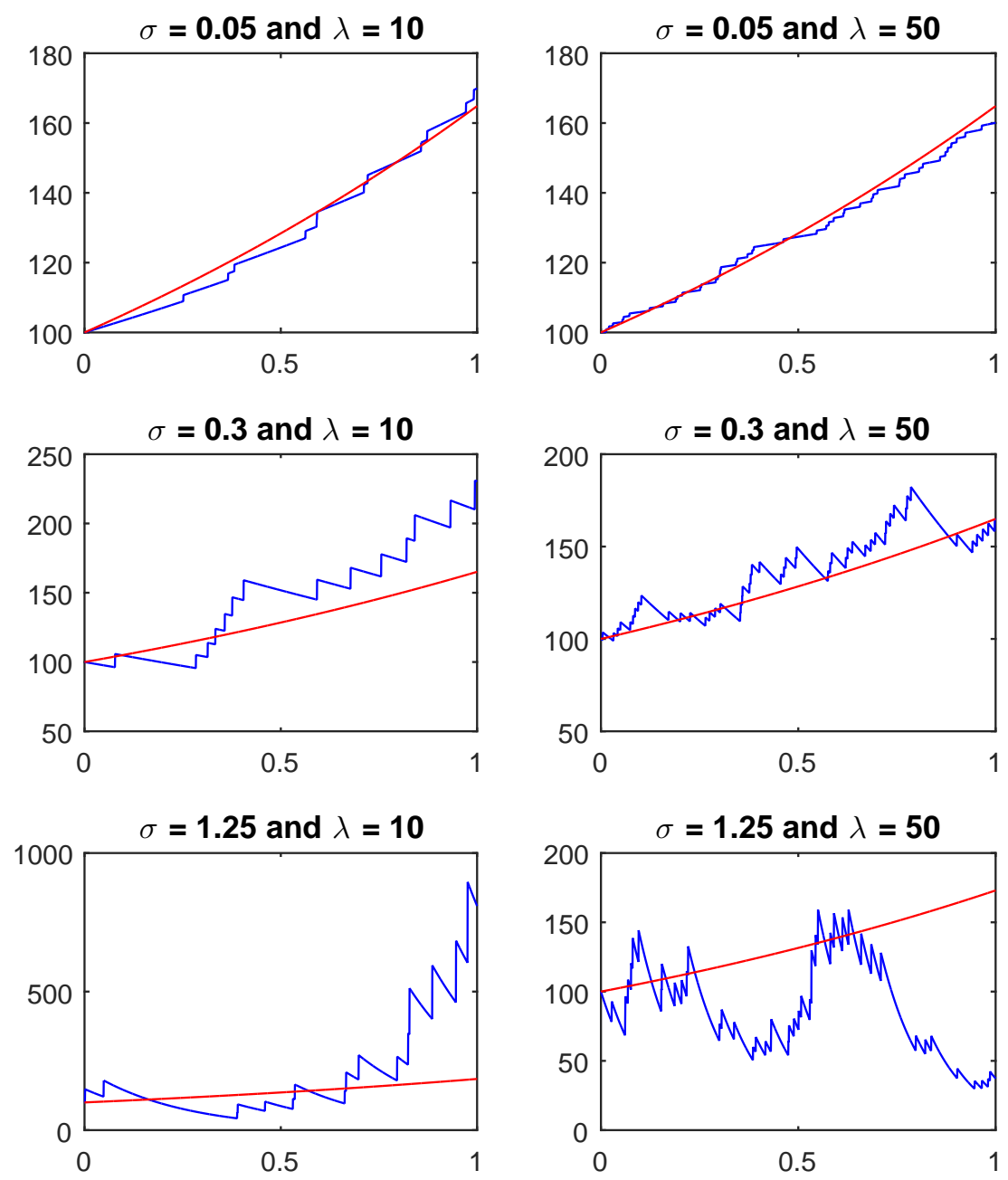

Figure 6: Trajectory of a CTMC stock (16) (blue lines) and respective mean functions (red lines), with $S_{0}=100, T=1, \alpha=0.5$. The values of $\lambda$ and $\sigma$ are written above each figure. 

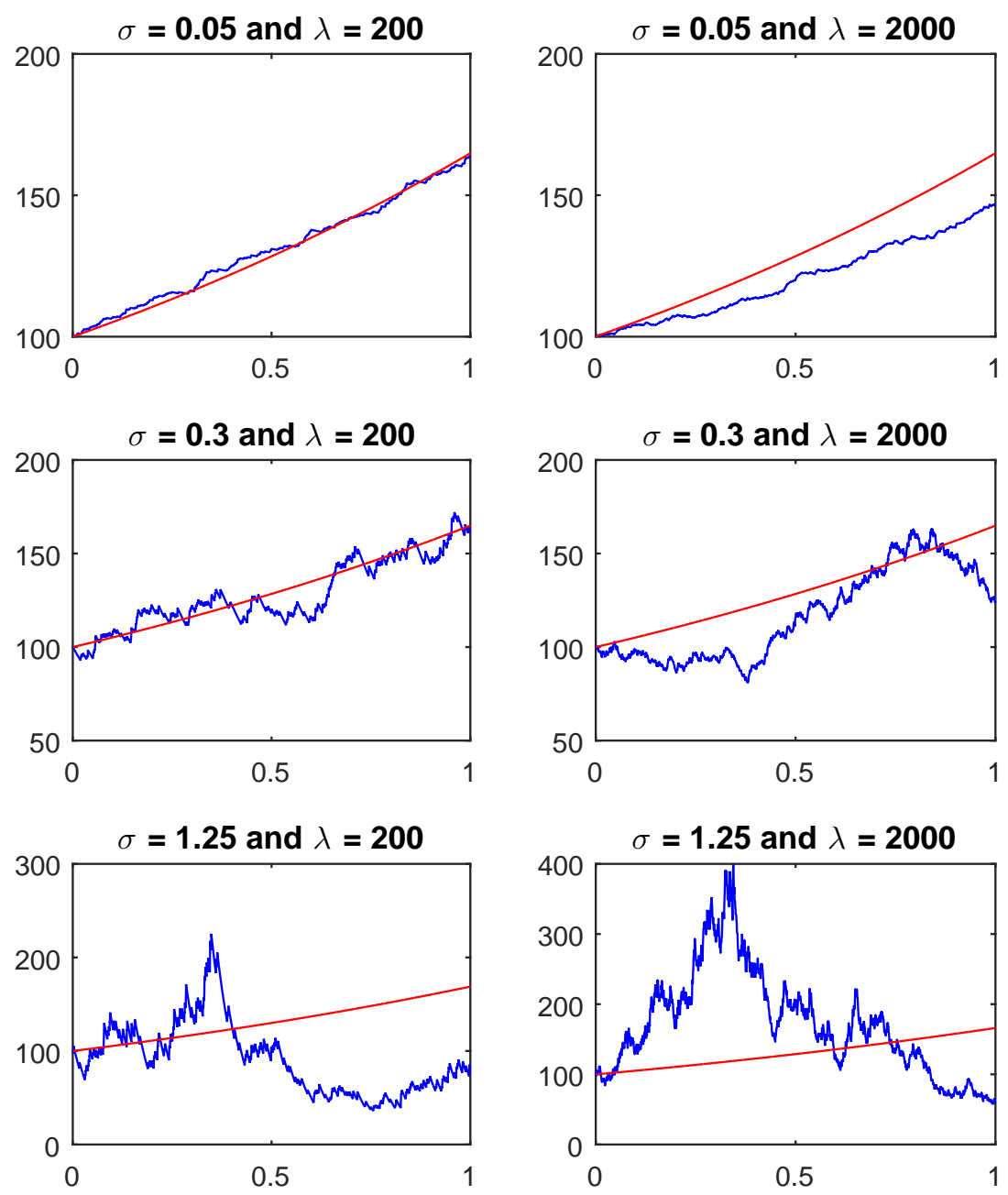

Figure 7: Trajectory of a CTMC stock (16) (blue lines) and respective mean functions (red lines), with $S_{0}=100, T=1, \alpha=0.5$. The values of $\lambda$ and $\sigma$ are written above each figure. 


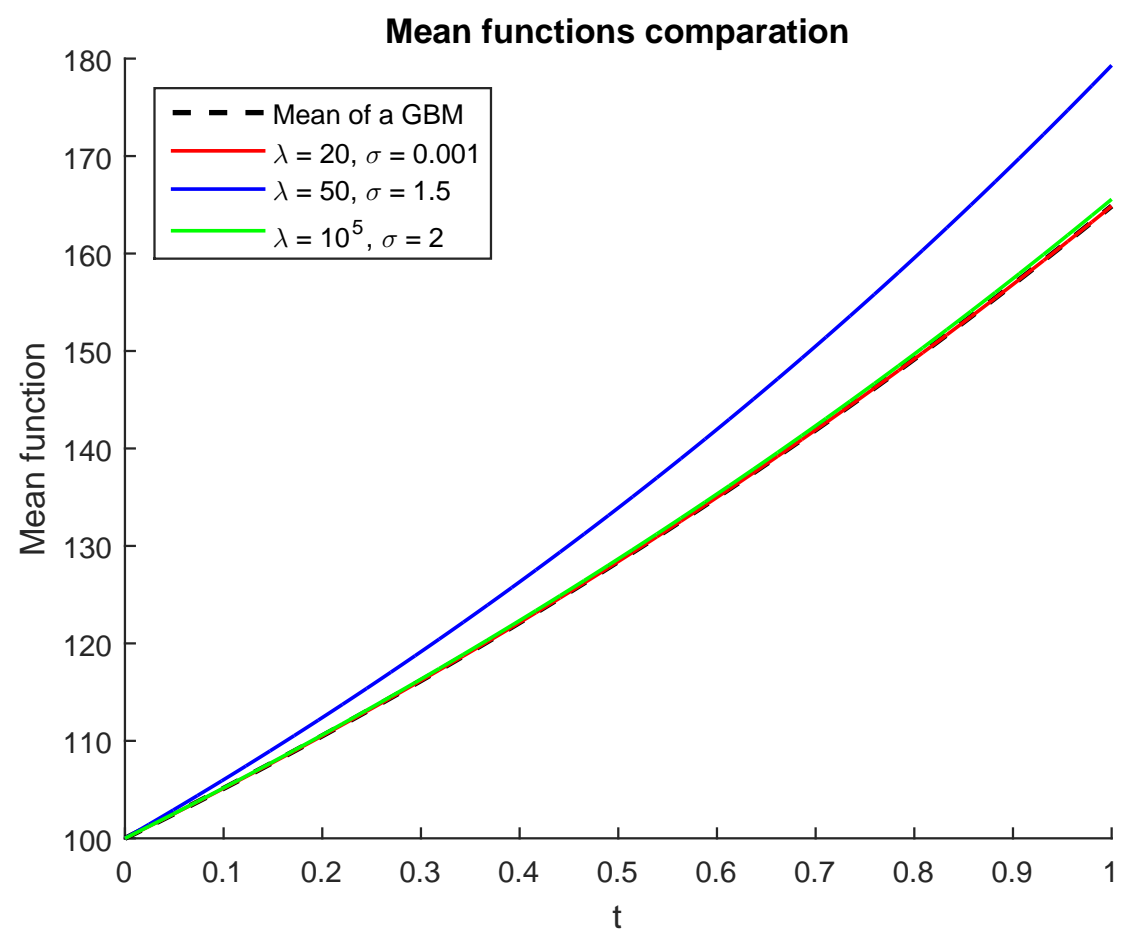

Figure 8: Plot of the mean function (24), with $S_{0}=100, T=1, \alpha=0.5$.

we have that this goes to eq. (14), as $\lambda \rightarrow+\infty$, which is a consequence of the following

$$
\begin{aligned}
\lim _{\lambda \rightarrow+\infty} \alpha & -\frac{1}{2} \sigma^{2}-\sigma \sqrt{\lambda}-\lambda+\lambda e^{\sigma / \sqrt{\lambda}}= \\
& =\lim _{\lambda \rightarrow+\infty} \alpha-\frac{1}{2} \sigma^{2}-\sigma \sqrt{\lambda}-\lambda+\lambda\left(1+\frac{\sigma}{\sqrt{\lambda}}+\frac{\sigma^{2}}{2 \lambda}+o\left(\lambda^{-3 / 2}\right)\right) \\
& =\alpha,
\end{aligned}
$$

for any $\sigma>0$. Moreover, we would also like to underline that the same result can be obtained taking $\sigma \rightarrow 0^{+}$, for any fixed $\lambda>0$. Heuristically, the latter appears to be rather obvious, since $\sigma$ represents the volatility of the model. In particular, we have

$$
\lim _{\sigma \rightarrow 0^{+}} \alpha-\frac{1}{2} \sigma^{2}-\sigma \sqrt{\lambda}-\lambda+\lambda e^{\sigma / \sqrt{\lambda}}=\alpha,
$$

as pointed out by the mean functions trajectory we have shown in Figure 8, 
where, for a fixed and non large value of $\lambda$, e.g., $\lambda=20$, and a small value of $\sigma$, e.g., $\sigma=0.001$, we have that the mean is almost equal to the one of a geometric Brownian motion, with the same parameter $\alpha$, etc. An analogues result holds taking a large value for $\lambda$, and a non small value for $\sigma$, e.g., $\lambda=10^{5}$, resp. $\sigma=2$. Conversely, a non small value of $\sigma$, and a non large value of $\lambda$, e.g., $\lambda=50$, resp. $\sigma=1.5$, give rise to opposite results.

\section{Conclusions}

In this work, we proved that the Norberg model provides a generalization of some well known models as the geometric Poisson model, the Black-Scholes model and the Merton model. In particular, we have proven that the last two proposals can be realised exploiting the weak convergence of a compensated Poisson process, normalized with respect to its intensity.

The high flexibility characterising the continuous-time Markov chain model (CTMC) is due to the scarse amount of a priori assumptions it needs, particularly concerning the distribution of the counting processes. Furthermore, since the state of the economy we are interested in, can be seen as a particular state of a CTMC, then the dependence of the assets price processes based on such an economy, turns to follow CTMC dependent dynamics.

Hence, latter approach allows to consider stock markets which take into account a rather ample and heterogeneous set of events such, e.g., recessions and booms, bullish and bearish trends, dependence between the assets, etc. Notice that we can also decide how to characterize the memory of the market model, thanks to the Markov property of the chain.

We would like to underline that a possible extension of the analysed Norberg model consists in considering time dependent transition intensities, $\lambda_{i j k}$, namely by considering an inhomogeneous CTMC. The latter proposal, which is actually one of our work in progress, will provide further generalisation of financial models different from the ones we studied in Sec. 4 as, e.g., for the case of stochastic volatility models.

Moreover, a further interesting generalisation consists in extending what we have reported in Sec. 4.3 to the general case where the random variables $A_{i}$ do not necessarily assume a discrete, finite, number of values, e.g., by considering a general type of Poisson measure, or a random perturbation of Lévy type.

We would like to recall that the CTMC theory can be successfully used as a powerful approach to the study of different subject in Finance. In particular, Norberg himself applies the CTMC approach in life insurance, see [24], and 
to arbitrage-free option pricing theory, see [26], where he also provides a nonstochastic partial differential equation for the price of a $T$-claim of the form $H=h\left(S_{T}\right)=\sum_{j=1}^{n} h_{j}\left(S_{T}\right)$, the index $j$ denoting the state of the Markov chain. Moreover, as a further development, the Norberg approach can be blended with the one related to asymptotic expansions technique, both in finite and infinite dimensional space, see, e.g., $[2,1]$ and references therein, allowing to obtain highly accurate approximations of some price functions which cannot be expressed in analytical form.

\section{References}

[1] S. Albeverio, F. Cordoni, L. Di Persio and G. Pellegrini, Asymptotic expansion for some local volatility models arising in finance, submitted (2016).

[2] S. Albeverio, L. Di Persio and E. Mastrogiacomo, Small noise asymptotic expansions for stochastic PDE's, I. the case of a dissipative polynomially bounded nonlinearity, Tohoku Mathematical Journal, 63, No. 4 (2011), 877-898.

[3] P.K. Andersen, O. Borgan, R.D. Gill and N. Keiding, Statistical models based on counting processes, Springer-Verlag, USA (1993).

[4] D.R. Baos, F. Cordoni, G. Di Nunno, L. Di Persio and E.E. Rse, Stochastic systems with memory and jumps, submitted, https://arxiv.org/abs/1603.00272 (2016).

[5] F. Beichelt, Stochastic processes in science, engineering and finance, CRC Press, (2006).

[6] C. Benazzoli and L. Di Persio, Default contagion in financial networks, International Journal of Mathematics and Computers in Simulation, 10, (2016), 112-117.

[7] T. Björk, Interest rate theory, Financial Mathematics, Springer (1997), 53-122.

[8] G. Bernis and S. Scotti, Alternative to beta coefficients in the context of diffusions, Quantitative Finance, (2016), 1-14.

[9] M. Bonollo, L. Di Persio and G. Pellegrini, Polynomial chaos expansion approach to interest rate models, Journal of Probability and Statistics, 5, (2015), 1-24.

[10] F. Cordoni and L. Di Persio, A BSDE with delayed generator approach to pricing under counterparty risk and collateralization, International Journal of Stochastic Analysis, (2016) .

[11] F. Cordoni and L. Di Persio, Invariant measure for the Vasicek interest rate model in the Heath-Jarrow-Morton-Musiela framework, Infinite Dimensional Analysis, Quantum Probability and Related Topics, 18, No. 3 (2015).

[12] F. Cordoni and L. Di Persio, Backward stochastic differential equations approach to hedging, option pricing, and insurance problems, International Journal of Stochastic Analysis, (2014).

[13] T. Björk, Arbitrage theory in continuous time, Oxford university press, USA (2009).

[14] F. Black and M. Scholes, The pricing of options and corporate liabilities, The journal of political economy, 81, No. 3 (1973), 637-654.

[15] P. Brémaud, Point processes and queues, Springer-Verlag, USA (1981). 
[16] L. Di Persio and M. Frigo, Gibbs sampling approach to regime switching analysis of financial time series, Journal of Computational and Applied Mathematics, No. 300 (2016), $43-55$.

[17] L. Di Persio,and M. Frigo, Maximum likelihood approach to Markov switching models WSEAS Transactions on Business and Economics, 12, (2015), 239-242.

[18] L. Di Persio and I. Perin, An ambit stochastic approach to pricing electricity forward contracts: The case of the German Energy Market,Journal of Probability and Statistics, (2015).

[19] L. Di Persio and G. Ziglio, Gaussian estimates on networks with applications to optimal control, Networks and Heterogeneous Media, 6, No. 2 (2011), 279-296.

[20] J. Jacod and A.N. Shiryaev, Limit theorems for stochastic processes, Springer-Verlag, Germany (2013).

[21] C. Marinelli, L. Di Persio, G. Ziglio, Approximation and convergence of solutions to semilinear stochastic evolution equations with jumps, Journal of Functional Analysis, 264, No. 12 (2013), 2784-2816.

[22] R.C. Merton, Lifetime portfolio selection under uncertainty: The continuous-time case, The review of Economics and Statistics, 51, No. 3 (1969), 247-257.

[23] R.C. Merton, Option pricing when underlying stock returns are discontinuous, Journal of financial economics, 3, No. 1-2 (1976), 125-144.

[24] R. Norberg, A time-continuous Markov chain interest model with applications to insurance, Applied Stochastic Models and Data Analysis, 11, No. 3 (1995), 245-256.

[25] R. Norberg, The Markov chain market, Astin Bulletin, 33, No. 2 (2003), 265-287.

[26] R. Norberg, Anomalous PDEs in Markov chains: domains of validity and numerical solutions, Finance and Stochastics, 9, No. 4 (2005), 519-537.

[27] F.A. Sonnenberg and J.R. Beck, Markov models in medical decision making: a practical guide, Medical decision making, 13, No. 4 (1993), 322-338.

[28] S.E. Shreve, Stochastic calculus for finance II: Continuous-time models, Springer-Verlag, USA (2004).

[29] D. Williams, Probability with martingales, Cambridge university press, Great Britain (1991). 